\title{
2 I 4 Temporal stability of the background velocity error shows potential for automated correction of regurgitant and shunt flow measurements
}

\author{
Peter D Gatehouse*, Nafisa Ajmal, Kilner J Philip, Dudley J Pennell and \\ David N Firmin
}

Address: Royal Brompton Hospital, London, UK

* Corresponding author

from I th Annual SCMR Scientific Sessions

Los Angeles, CA, USA. I-3 February 2008

Published: 22 October 2008

Journal of Cardiovascular Magnetic Resonance 2008, I0(Suppl I):A383 doi:I0.I I86/I532-429X-I0-SI-A383

This abstract is available from: http://jcmr-online.com/content/I0/SI/A383

(c) 2008 Gatehouse et al; licensee BioMed Central Ltd.

\section{Introduction}

It is well-known that velocity images can show a background offset in stationary tissue. Although the offset is reduced after correcting concomitant gradients, it has recently been shown [1] that applications such as the assessment of regurgitation or shunt flow are extremely sensitive to the residual error. The background offset usually varies across the image and is hard to predict, being dependent on numerous sequence parameters. Background errors even smaller than $1 \%$ of the VENC can seriously affect measurements of regurgitant fraction. Correction depends on obtaining a background error image for subtraction from the in-vivo velocity image. Accuracy is improved by velocity imaging of a stationary phantom acquired with identical sequence parameters after the patient scan [1]. However, this procedure may be impracticable for clinical work.

This abstract therefore investigates the feasibility of using corrections acquired once after service recalibration, where the correction image nearest to that of the patient is used to remove the error. We present work on two main difficulties with the stored-corrections idea: First, the set would require thousands of images, covering all angles, slice offsets, inplane rotations, slice thicknesses, spatial and temporal resolutions, VENCs etc.; impracticable to acquire, so its size must be reduced as proposed below. Second, it assumes that the background error is stable over time.

\section{Purpose}

To improve accuracy of flow MRI by automatic correction using stored background velocity errors. Fundamental to this idea, we first assess how stable is the background offset error in velocity images over a period of several weeks.

\section{Methods}

For regurgitant fraction or shunt flow, only planes transecting the aorta and pulmonary trunk are used which typically lie at about $45^{\circ}$ to transaxial planes as shown in Figure 1 . The size of the phantom data sets is reduced by including only a limited range of angles, offsets and inplane rotations, sufficient to cover the AO and PA planes of most patients. First, to evaluate temporal stability, the $\mathrm{AO}$ and PA planes as shown in Figure 1 were each acquired for -50, -25, 0, 25, $50 \mathrm{~mm}$ offsets along the headfoot direction, and the PA plane was repeated with alternate phase-encode direction. The set of 15 planes was reacquired over 40 days. For assessment of errors down to $0.25 \%$ of VENC, a gelatin phantom prevented flow, doped with copper sulphate enabling higher velocity-tonoise ratio. Only retro-gated cine imaging sequence was used, as in patient studies (at $1.5 \mathrm{~T}$ Siemens Avanto), because the steady gradient activity improved cine frameto-frame background stability.

\section{Results and discussion}

The background errors were found to vary linearly over the image, with a constant offset. Fitted slope and offset 


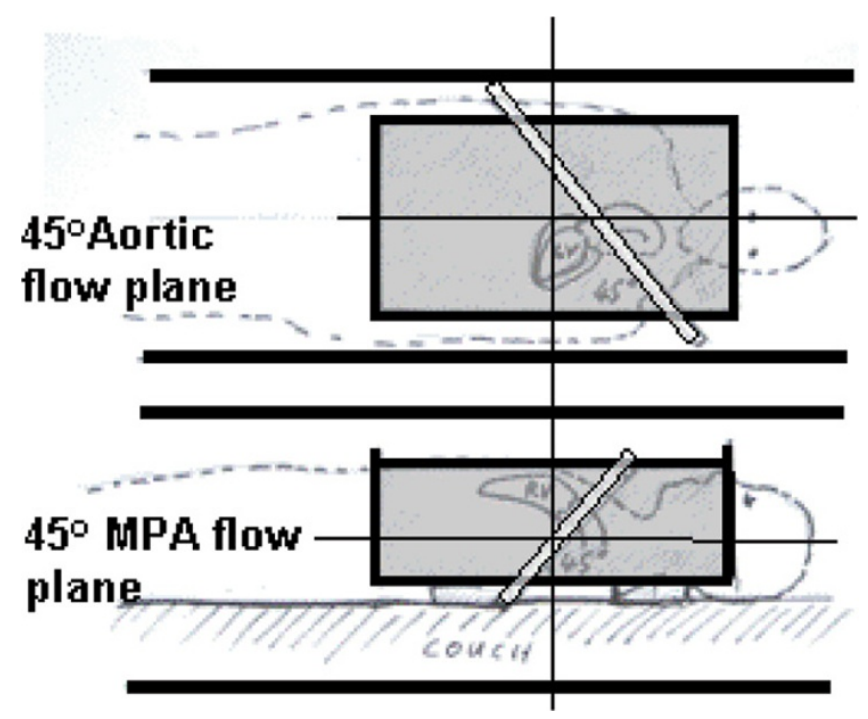

Figure I

The aortic and MPA planes where highest accuracy is required for regurgitant and shunt flow. The gray box represents the gelatin phantom.

values for the 15 planes over 40 days (Figure 2) demonstrated the stability of the background error over time. Recalibration of eddy-current compensation (before and after the time plotted on Figure 2) sometimes caused large changes. In between such recalibrations, the near-constant helium level (especially of this magnet type with helium recirculation) appears to maintain the stability of eddy-currents. Only the fitted parameters need be stored, and the correction can be performed without reducing the velocity-to-noise ratio in the velocity image. Some sequence parameters such as phase-encode field-of-view do not affect the background, but the database would have to support a range of other parameters. The restricted range of planes, to those where this accuracy is needed, makes the method more feasible.

\section{Conclusion}

The stability of the background error over 40 days has been demonstrated. We are not aware that this has been shown before. It supports the possible use of a stored set of background corrections for improved accuracy of regurgitant and shunt flow measurements. 

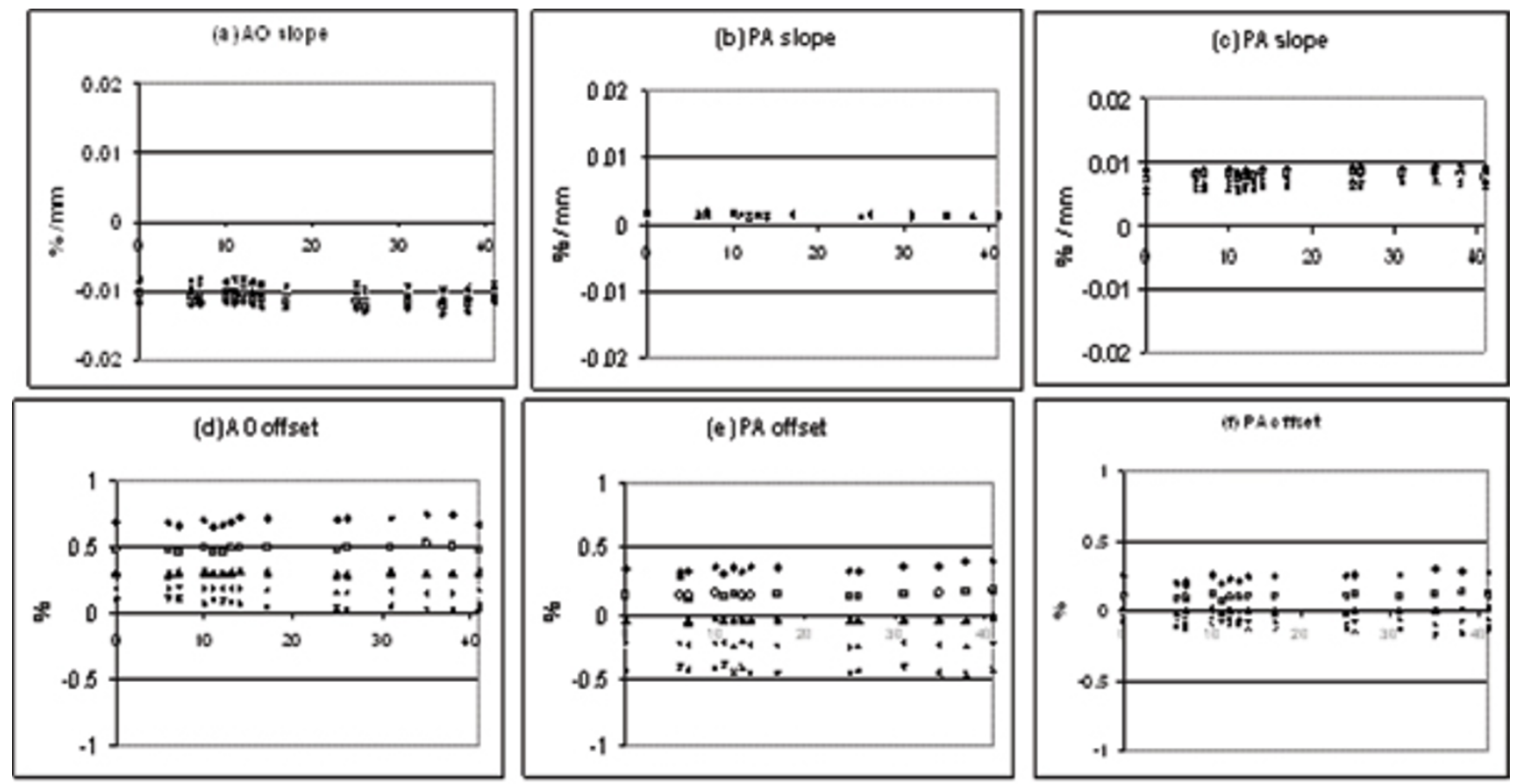

Figure 2: The horizontal axis in each graph is the 40-days of repeated background error studies at the five offsets $(-50 \ldots 50 \mathrm{~mm}) . \mathrm{AO}=$ aortic plane. $\mathrm{PA}=$ main pulmonary artery plane.

sones

(a-c) Slope in background error (\% of VENC per mm along FE-axis).

(d-f) Shift in background error at image centre (\% of VENC).

\section{Figure 2}

The horizontal axis in each graph is the 40 days of repeated background error studies at the five offsets $(-50 \ldots 50 \mathrm{~mm}) . \mathrm{AO}=$ aortic plane. PA = main pulmonary artery plane. $(a-c)$ Slope in background error (\% of VENC per mm along FE axis). (d-f) Shift in background error at image centre (\% of VENC).

\section{References}

I. Chernobelsky, Shubayev, Comeau, Wolff : JCMR 2007, 9:68I-5. 